\title{
La Necesidad de Aplicar el Método Científico en Investigación Clínica. Problemas, Beneficios y Factibilidad del Desarrollo de Protocolos de Investigación
}

\author{
The Scientific Method. Its Relevance in Conducting Clinical Research
}

\author{
Tamara Otzen ${ }^{1,2}$; Carlos Manterola ${ }^{1}$; Iván Rodríguez-Núñez ${ }^{3} \&$ Maricela García-Domínguez ${ }^{4}$
}

OTZEN, T.; MANTEROLA, C.; RODRÍGUEZ-NÚÑEZ, I. \& GARCÍA-DOMÍNGUEZ, M. La necesidad de aplicar el método científico en investigación clínica. Problemas, beneficios y factibilidad del desarrollo de protocolos de investigación. Int. J. Morphol., 35(3):1031-1036, 2017.

RESUMEN: La metodología de la investigación científica constituye un conjunto de métodos, leyes y procedimientos que orientan los esfuerzos de la investigación hacia la solución de los problemas científicos con un máximo de eficiencia. El método se basa en el desarrollo de distintas etapas que conducen al establecimiento de una conclusión válida sustentada en la verificación de una hipótesis y supuestos de la investigación realizada. Así, la investigación científica no está exenta a la ocurrencia de errores, los cuales pueden ser originados durante el planteamiento del problema de estudio, el diseño de investigación, el establecimiento de los criterios de elegibilidad, la estimación del tamaño de la muestra, el proceso de medición, durante el seguimiento de los sujetos o debido a la falta de discusión de las limitaciones del estudio. En este contexto, una de las estrategias que permite reducir los potenciales errores en la conducción de un estudio radica en el adecuado desarrollo de un proyecto o protocolo de investigación, el cual, debe ser elaborado en base a un problema de investigación, diseño y método de medición, otorgándole al investigador una serie de ventajas asociadas con la planificación documentada del proceso, reproducibilidad de los protocolos experimentales y facilitación del proceso de divulgación de los resultados. En la presente revisión se discuten los aspectos esenciales referentes al método científico en investigación clínica y se analizan los principales problemas, beneficios y elementos asociados con la factibilidad de desarrollar un protocolo de investigación.

PALABRAS CLAVE: Investigación clínica; Método científico; Diseños de investigación; Metodología de la investigación.

\section{INTRODUCCIÓN}

La ciencia es un sistema de conocimientos sobre la realidad que nos rodea. Un sistema que abarca leyes, teorías e hipótesis; que se encuentra en un proceso continuo de desarrollo, lo que representa el perfeccionamiento continuo del conocimiento sobre la realidad actual, pasada y de cierta forma la futura. La metodología de la investigación científica constituye por su parte un conjunto de métodos, leyes y procedimientos que orientan los esfuerzos de la investigación hacia la solución de los problemas científicos con un máximo de eficiencia. Se trata pues, de la aplicación del método científico en la solución de problemas del conocimiento (Jiménez, 1998).

La historia de la ciencia en la medicina comenzó en 1543 con el libro de Vesalio "De humani corporis fabrica libri septem"; considerado uno de los más prestigiosos li- bros científicos de todos los tiempos, basado en las conferencias que el autor dictó en la Universidad de Padua. Cuatrocientos años más tarde, en 1955, Braun extendió el basto e inexplorado territorio de la medicina aplicada para futuras investigaciones científicas con su publicación "La ley de la distribución regular de casos"; obra en la que la tarea más relevante fue divulgar las leyes inherentes y crear una terminología concreta y adecuada (Temml, 2001).

Por ende, se ha de tener claro que el método científico, constituye la columna vertebral de cualquier proceso de investigación, caracterizándose por una serie de etapas que, observadas y seguidas de forma acuciosa y sistematizada; permiten conducir y concluir cualquier protocolo de investigación (Fig. 1).

\footnotetext{
${ }^{1}$ Centro de Excelencia en estudios Morfológicos y Quirúrgicos (CEMyQ), Universidad de La Frontera, Temuco, Chile.

${ }^{2}$ Facultad de Ciencias de la Salud, Universidad de Tarapacá, Arica, Chile.

${ }^{3}$ Escuela de Kinesiología, Facultad de Ciencias de la Salud, Universidad San Sebastián, Concepción, Chile.

${ }^{4}$ Universidad Nacional Autónoma de México, Unidad León, México.
} 


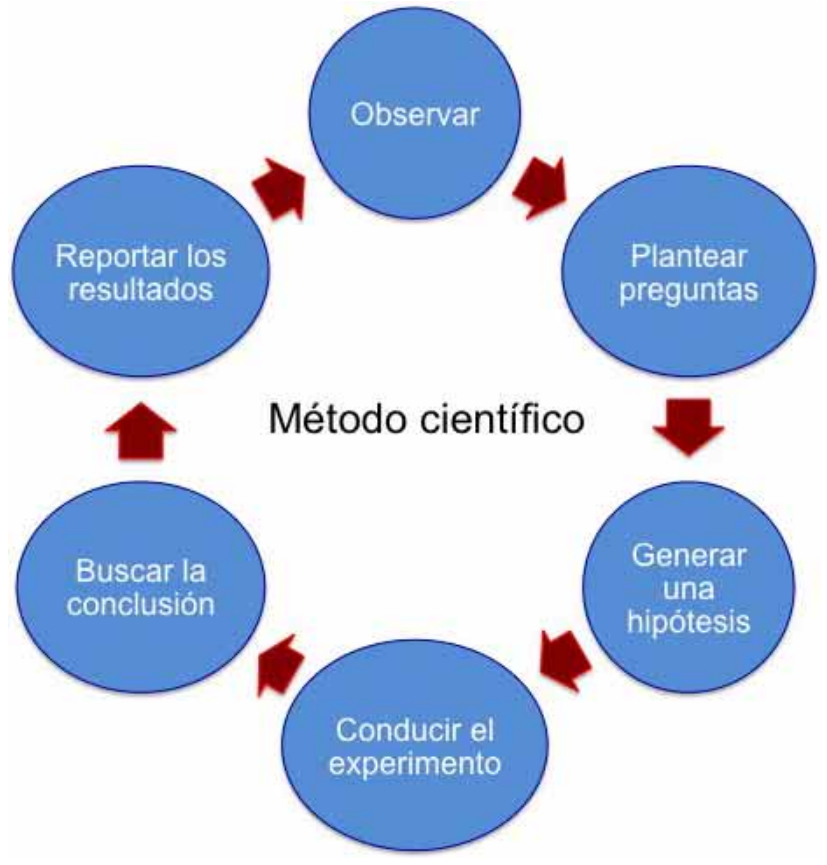

Fig. 1. Descripción resumida del método científico.

El esfuerzo de la investigación clínica (IC) para estudiar a un grupo de pacientes es hacer un diagnóstico, estimar el pronóstico y sugerir un tratamiento; para lo cual, es fundamental la utilización del método científico (MC) (Rubino, 1988): que se puede resumir en la regla arquitectónica que a su vez se puede dividir en causa-efecto y el proceso de investigación; el enfoque metodológico, que incluye cualquier tipo de diseños de estudios, como ensayos clínicos (EC), revisiones sistemáticas (RS), estudios de cohortes, de casos y controles, etc. (Rubino; Lader et al., 2004; Hartung \& Touchette, 2009; Manterola \& Otzen, 2014), todos los que se desarrollan siguiendo las etapas del método científico (Fig. 2).

Por otra parte, los tipos de estudios señalados anteriormente, pueden ser utilizados para responder interrogantes correspondientes a escenarios sobre terapia o procedimientos terapéuticos, pronóstico e historia natural, pruebas diagnósticas, etc. Todos estos, se agrupan en el concepto de estudios primarios, es decir, que los datos se obtuvieron directamente a partir de los sujetos estudiados puesto que existe una segunda categoría de estudios; denominados "estudios secundarios o integradores", en los que los datos son obtenidos a partir de estudios primarios (Talavera, 2011; Sessler \& Imrey, 2015).

De este modo, es que el paradigma de la medicina basada en la evidencia (MBE), propone el uso de la mejor evidencia disponible en el momento, para la toma de decisiones en salud; de modo tal, que estas sean correctas y bien informadas; lo que no significa objetar de forma instantánea la evidencia denominada de "baja calidad", así como tampoco aceptar los EC con asignación aleatoria, como la única fuente de verdad y de evidencia irrefutable (Lader et al.).

Así, para realizar una recomendación respecto de la mejor evidencia disponible, es esencial realizar una búsqueda exhaustiva de la literatura, valorando elementos metodológicos fundamentales como el diseño del estudio, su calidad metodológica (validez interna), la magnitud de sus resultados, y la reproducibilidad de estos en diferentes poblaciones (validez externa) (Manterola et al., 2014).

En esta historia de la IC, se desarrolló de forma casi simultánea a la MBE, el concepto de "clinimetría", término introducido por Alvan Feinstein, Matemático y Médico Norteamericano, a principios de los años ochenta; para indicar un ámbito relacionado con índices, escalas de calificación y otras expresiones utilizadas para describir o medir síntomas, signos físicos y otros fenómenos clínicos. Por ende, la clinimetría es un conjunto de reglas que rigen la estructura de los índices, la elección de las variables componentes, la evaluación de la consistencia, la validez y la capacidad de respuesta. De este modo, se ocupa de los métodos cuantitativos en la recopilación y análisis de datos clínicos comparativos y, en particular, con la mejora en los procesos de "medición" de los fenómenos clínicos y personales distintivos de la atención al paciente (Feinstein, 1983; Fava et al., 2012). La ciencia clínimétrica ofrece oportunidades para generar nuevos enfoques ó un tipo adicional de IC que puede incrementar la base científica de la práctica clínica, humanizando el contenido de los datos de investigación y restableciendo el énfasis analítico en el arte del cuidado del paciente (Tomba \& Bech, 2012).

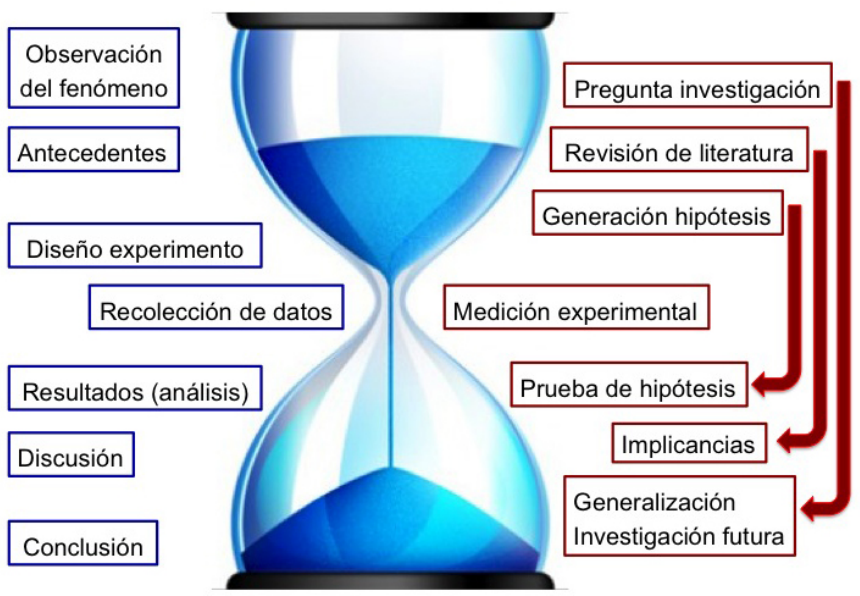

Fig. 2: Etapas del método científico. 
Sin embargo, es fundamental comprender que la IC es un proceso por medio del cual, se pretende dar respuestas. Se caracteriza por ser sistemático, porque a partir de un objetivo se recogen datos de acuerdo a un protocolo preestablecido que, luego serán analizados e interpretados, aportando nuevos conocimientos a los ya existentes; organizado, debido a que todos los miembros del equipo conocen lo que hay que hacer durante todo el estudio y actúan de igual forma ante los escenarios que correspondan; y objetivo, ya que las conclusiones obtenidas se basan en hechos observados y medidos, y no en impresiones subjetivas.

\section{PROBLEMAS Y ERRORES MÁS COMUNES EN IC}

A continuación, se enumerarán los descuidos y omisiones más frecuentes de observar en la conducción de la IC: todo comienza habitualmente por el principio, por no darse el tiempo suficiente para plantear una pregunta de investigación y por ende un objetivo; e idealmente, la justificación de la investigación, es decir, explicar porque es conveniente llevar a cabo ésta investigación, prediciendo los beneficios que ésta puede reportar (Manterola \& Otzen, 2013). A partir de este error, se desprende una serie de otros que se comentarán a continuación: No desarrollar un protocolo o proyecto de investigación; no examinar cuidadosamente la literatura existente respecto de investigaciones similares anteriores; es decir, no construir o construir de forma defectuosa el marco teórico, que constituye el marco de referencia para la elaboración de un proyecto y posteriormente para la publicación de los resultados; enriqueciendo además los conocimientos que poseemos sobre el tema de investigación, orientándose sobre como debe realizarse; y ayudando a prevenir más errores. Otra desprolijidad, tiene que ver con no aplicar una evaluación crítica de la literatura existente (Clark \& Mulligan, 2011), lo que nos puede llevar a subestimar publicaciones que pueden ser un aporte a la conducción del estudio; o por el contrario, a sobrestimar y sobrevalorar algunas carentes de validez interna y externa.

La elección del diseño para dar respuesta a la pregunta de investigación más eficiente, es sin duda un desafío, puesto que entre otras cosas, pone en jaque la factibilidad del proceso porque se relaciona directamente con costes, nivel de experiencia, infraestructura, etc. (Manterola \& Otzen, 2014).

Otras etapas fundamentales de este proceso, tienen que ver con la desprolijidad en la determinación de los criterios de inclusión y exclusión de los sujetos a estudio. La definición, selección y representatividad de la muestra con la que se llevará a efecto el estudio; y que en rigor, debe ser representativa de toda la población que le dio su origen, de lo contrario, queda en riesgo la posibilidad de extrapolar los resultados o de cometer uno de los sesgos más comunes, que es el sesgo de selección (Manterola \& Otzen, 2015).
Asociado a esto, aparecen otros potenciales problemas, como no estimar el tamaño de la muestra antes de comenzar el estudio, o estimarla de forma errónea. Sin embargo, en situaciones en las que este paso no es posible, se ha de tener en consideración que el tamaño mínimo para conducir una IC debe ser en lo posible superior a 100 (Manterola \& Otzen, 2013).

Luego, aparecen otros potenciales problemas como son: fallas en la implementación de medidas de control de sesgos, reclutamiento inadecuado de participantes ó retención apropiada de los sujetos a estudio que fueron reclutados. Errores en el proceso de medición, lo que conduce a sesgo de medición (Clark \& Mulligan; Manterola \& Otzen, 2015). No examinar la normalidad de los datos y error en la diferenciación de los supuestos estadísticos utilizados en el análisis de los datos.

A la hora de reportar los resultados, pueden aparecer otros problemas, como no reportar datos faltantes, sujetos que abandonaron el seguimiento (o que no lo completaron); así como no realizar un análisis con intención de tratar; o no reportar medidas de efecto como incidencia de riesgos, número necesario a tratar o dañar y sus respectivos intervalos de confianza del $95 \%$, etc.

Finalmente, a la hora de escribir la discusión, quizás el error más notable tenga relación con no señalar de forma explícita las debilidades o limitaciones de su propio estudio (Manterola \& Otzen, 2017).

\section{EL PROYECTO O PROTOCOLO DE INVESTIGACIÓN}

No es otra cosa que el documento donde se plasman por escrito todos los detalles de la planificación de la investigación; es decir, qué se investiga, por qué y sobre todo cómo.

En términos generales, se reconoce que un proyecto de investigación debe contener los siguientes elementos: La introducción o presentación general del problema a investigar, focalizando los puntos clave de la investigación. El marco teórico, con una evaluación crítica de la información existente, de cuyo análisis surge la justificación definitiva para el estudio propuesto. La justificación de la investigación, que consiste en una fundamentación de la importancia de ejecutarla, mencionando los aspectos relevantes de ésta y sus potenciales implicaciones futuras. La metodología de la investigación que se utilizará en la conducción del estudio, que involucra: la pregunta de investigación, la hipótesis, los objetivos, el diseño y descripción general del diseño a utilizar; la muestra (población de interés, descripción de la muestra, cálculo del tamaño de la muestra, métodos de muestreo, criterios de elegibilidad, etc.); descripción de la intervención, exposición o evento a estudiar; proceso de medición de la(s) 
variable(s); el plan de análisis (estadísticas a emplear según los resultados que se espera); una carta Gantt; aspectos éticos y económicos o de financiamiento involucrados en la investigación. Finalmente, se deben considerar las referencias bibliográficas.

Sin embargo, un hito fundamental lo constituye la pregunta de investigación que además de cumplir con los criterios FINER (factible, interesante, novedosa, ética y relevante) debe tener un sustento en el potencial beneficios de los pacientes. Se recomienda plantearla utilizando el acrónimo PICO (PICoR) o PECO (dependiendo si se trata de una intervención o de una exposición), Incluye los elementos que debe contener una pregunta de investigación clínica. Por ejemplo, ante la pregunta ¿Cuál será la supervivencia libre de enfermedad en pacientes con cáncer colorectal utilizando cirugía versus quimioterapia con anticuerpos monoclonales?, P, serán los individuos con cáncer colorectal; I será cirugía; C (Co), será quimioterapia con anticuerpos monoclonales; y $\mathrm{O}(\mathrm{R})$, será supervivencia libre de enfermedad. De este modo, se pueden realizar incluso las búsquedas de información científica en metabuscadores como Trip Database, que al aplicar estos mismos términos en su opción de búsqueda PICO, con la estrategia "(Colorectal Neoplasm) (Surgery) (Chemotherapy with monoclonal antibody) (Disease-Free-Survival)", genera (al 2 de mayo de 2017), un total de 439 resultados, agrupados por tipo de diseño, nivel de evidencia, etc.

El ejecutar este tipo de acciones y contar con este documento, otorga una serie de ventajas para el investigador. Permite tener la planificación documentada, sistematizada de forma consciente y no sujeta a la memoria. Permite que otro(s) investigador(es), puedan ejecutar el protocolo en el caso que el investigador responsable no pudiera continuar haciéndose cargo del mismo. Y por último, facilita el proceso de publicación y de presentación de resultados posteriores en congresos, pues estará toda la información ya redactada de forma organizada.

\section{¿QUÉ BENEFICIOS OBTIENE UN CLÍNICO AL HA- CER INVESTIGACIÓN?}

A continuación, se enumerarán algunos de los beneficios más destacables que puede esperar un clínico que hace investigación. Primero, hay que destacar que la necesidad de realizar una continua actualización de la bibliografía, determina que el clínico se mantendrá sus conocimientos al día, lo que de forma indirecta, un aumento de su eficiencia en la práctica clínica. Esto, va directamente aparejado con una mejor forma de enfrentarse a los problemas clínicos cotidianos.

En segundo lugar, es importante señalar que la necesidad de tener que centrarse en un problema de investiga- ción concreto, determina que se pueden poner a punto una serie de técnicas y procedimientos que ayudan al proceso diagnóstico-terapéutico del problema de investigación.

Finalmente genera el beneficio de ir incrementando de forma continua y paulatina la creatividad, ya que, el hecho de romper la rutina incrementa la motivación. Sin embargo, hay una serie de consideraciones que se deben tomar en cuenta respecto de la IC. Por ejemplo, comprender que el hecho que un autor tenga prestigio por su experiencia en la práctica profesional, NO es garantía de validez y de calidad de su publicación; debido a que el proceso de investigación requiere de una preparación rigurosa en metodología, lo que no tiene relación con la práctica profesional. $\mathrm{O}$, que el contenido de una publicación es válido porque la información procede de una "fuente responsable o capacitada" (todo lo que se publica en la revista x es cierto"); sin embargo, existe evidencia respecto de que el interés de muchas investigaciones científicas es exiguo, limitado o inexistente; que existe ambigüedad y confusión de datos, incluso que el fraude es más frecuente de lo que se piensa; que parte de lo publicado es científicamente erróneo o metodológicamente inválido; y que determinados artículos científicos de interés no llegan a ser publicados, o se publican en revistas de escaso visibilidad. Así también, se tiende a pensar que el hecho que la investigación publicada utilice como diseños un EC o una RS constituye garantía de validez científica; pero, se ha de recordar que la conducción de este tipo de estudios representa dificultades metodológicas que lo hacen siempre complejo, por ende, no todos los EC y RS son lo que pretenden ser (sesgos y errores metodológicos y éticos terminan debilitándolos o invalidándolos). Es fundamental considerar el valor y limitaciones de los estudios observacionales, herramienta de gran utilidad en IC y epidemiológica (Choh \& Priolo, 1999; Hartung \& Touchette; Manterola \& Otzen, 2014).

Algo similar puede esperarse si es que un autor ya publicó un artículo científico de buena calidad metodológica, tampoco es garantía suficiente de calidad de su nueva publicación; a lo más podrá esperarse una mayor probabilidad de calidad. Un concepto interesante es que, al menos, parte del prestigio de los profesionales sanitarios se basan en el número de artículos científicos publicados; incluso más que en la calidad que éstos puedan tener (de Rovira \& Tapia Granados, 1995).

Por otro lado, se piensa que el número de publicaciones científicas es demasiado grande para mantenerse al día dado el escaso tiempo disponible; sin embargo, el acceso informatizado a metabuscadores, bibliotecas y bases de datos, como por ejemplo Trip Database, BIREME o BVS, SciELO, PubMed, EMBASE, etc., permite obtener una búsqueda bibliográfica relativamente simple, rápida, actualizada y completa. 
OTZEN, T.; MANTEROLA, C.; RODRÍGUEZ-NÚÑEZ, I. \& GARCÍA-DOMíNGUEZ, M. La necesidad de aplicar el método científico en investigación clínica. Problemas, beneficios y factibilidad del desarrollo de protocolos de investigación. Int. J. Morphol., 35(3):1031-1036, 2017.

\section{FACTIBILIDAD Y PERTINENCIA DE LA IC}

Entre los criterios que pueden darle factibilidad a una investigación, se pueden enumerar: tener buenas ideas, disponer de tiempo y dedicación para la ejecución del proyecto; contar con cierto nivel de experiencia en investigación y de conocimientos (al menos elementales) en metodología de investigación.

A estos principios, se pueden sumar el poder contar con apoyo de personal con habilidades en la utilización de programas informáticos, con experiencia en metodología de investigación y bioestadística; y de la colaboración con otros investigadores, equipos o centros de mayor experiencia. Esto, sin lugar a dudas, es fundamental para el tratamiento de datos y uso de cierto tipo de variables medibles y cuantificables.

Si se trata de un proyecto de IC, es muy relevante poder disponer de un número suficiente de pacientes o sujetos a estudio, en los que deben ser observadas las consideraciones éticas de la investigación con seres humanos. Y, contar con los recursos económicos adecuados para costear el proyecto de investigación.

En referencia a la pertinencia de ejecutar una IC, deben ser considerados algunos criterios como que la pregunta de investigación planteada no ha sido contestada, se contestó de forma contradictoria o requiere adecuación a la práctica clínica del medio en que nos desenvolvemos (lo mismo para el objetivo de la investigación). Por otra parte, está la trascendencia del problema que se quiere investigar respecto de un evento de interés, en términos de su frecuencia, gravedad, interés social, importancia de su valor teórico y de eventuales beneficios que puedan derivarse de los resultados de la investigación propuesta (Pita Fernández, 1999).

\section{LA ESTADÍSTICA.}

La Punta Visible del Iceberg de la Metodología de Investigación. La estadística es una disciplina que se vale de instrumentos científicos que requieren del cumplimiento de numerosas condiciones para producir resultados válidos. Por un lado, es preciso que los estudios tengan un diseño muy estricto. Y por otro, es injustificable aplicar en cada circunstancia aquellos métodos que en ella son idóneos y controlar rigurosamente su validez. Pero ni aún así es legítimo deducir que un resultado significativo es clínicamente relevante. Cabe recordar que una correlación entre dos variables acabará siendo siempre significativa $(\mathrm{p}<0,05)$ si la muestra es lo suficientemente grande (Altman, 2000). Por eso y en este contexto, alguien comentó: "la estadística es un instrumento, que si no se usa adecuadamente, puede demostrar cualquier mentira".
Muchos investigadores tienen bien definido su estudio y utilizan de forma adecuada los datos y variables, pero no aciertan cuando escogen las pruebas estadísticas pertinentes, y llegan a la redacción del artículo, con un manuscrito que adolece de forma ostensible de sincronía entre datos y su ulterior análisis estadístico (Antoja Ribó, 2010).

Al respecto es menester señalar que un artículo científico en el que se demuestran ventajas de una intervención en estudio respecto de la intervención habitual, con un valor de $p<0,05$ ó "estadísticamente significativo"; la mayor parte de los lectores suelen interpretar que el resultado es importante y por ende debe tener significación clínica. Pero, se ha de considerar que, si bien, es cierto que algunos resultados estadísticamente significativos pueden ser de gran utilidad en la práctica clínica, el "valor de p", debe ser valorado con precaución, tomando en consideración el contexto del estudio, su diseño, las características de la muestra analizada, la existencia de los potenciales sesgos, etc. Y jamás como una cifra mágica que nos embobe al punto de llevarnos a tomar decisiones o cambiar conductas ya probadas (Altman). Por lo anteriormente expuesto, es que antes de tomar decisiones o de cambiar conductas clínicas basadas en un "valor de p", se ha de considerar la validez externa de los resultados del estudio; ó la real posibilidad de generalizar o extrapolar los resultados a nuestros pacientes o nuestra realidad, la que puede ser muy diferente a la del estudio publicado (Manterola et al., 2008). Es decir, poder diferenciar entre lo estadísticamente significativo a lo clínicamente relevante; debido a que los hallazgos estadísticos derivan de grupos de sujetos, requieren de un tamaño de muestra adecuado para ser válidos; $\mathrm{y}$ a pesar que pueden reflejar la influencia del azar en los resultados, hay que recordar que se trata de respuestas probabilísticas (Altman). Por otro lado los hallazgos clínicos provienen de individuos específicos, suelen reflejar el valor biológico del resultado, y que las decisiones clínicas se toman con alguna frecuencia con datos insuficientes; por lo tanto, las terapias y procedimientos terapéuticos requieren decisiones oportunas comprometidas; y que la práctica clínica usualmente no puede medir cada función, sistema, o manifestación clínica. Por lo que resulta más apropiado hablar de "relevancia clínica" que de "significación estadística"; porque la relevancia clínica de un fenómeno va más allá de cálculos matemáticos y depende efectivamente de la gravedad del problema, morbilidad y mortalidad generada por el mismo, magnitud de la diferencia entre dos tratamientos, costes involucrados, etc.

Es así como un resultado "estadísticamente significativo" sin relevancia clínica no deja de ser una anécdota; así como un hallazgo clínicamente importante sin significación clínica, no puede ser asumido como concluyente, ya que no podemos atribuir con seguridad el hallazgo clínico a 
la intervención realizada (influencia del azar). La literatura científica viene abusando de la "significación estadística", y es así como muchos investigadores dan por supuesto que si en un estudio hallan una probabilidad inferior a 0,05 ; esto cerciora la existencia de validez de los resultados, así como la relevancia clínica de los mismos (Pocock, 1980; Manterola et al., 2008). La estadística es una herramienta útil para la realización de estudios clínicos, pero debe ser utilizada de forma correcta.

La utilidad del valor de p es simplemente una medida de la influencia del azar (el que debe ser advertido siempre, y cuantificado a través del valor de p), en la posible asociación entre las variable analizadas, no es un indicador de fuerza de la asociación entre variables, ni de su importancia; es así como sus valores deben ser considerados sólo como una guía y no como base de conclusiones definitivas e irrevocables (Altaman, 2000; Manterola et al., 2008).

y... ¿qué es lo que significa un valor de $\mathrm{p}<0,05$ ? Solamente que en menos de 5 de cada 100 veces que repitiéramos el mismo estudio, nuestro resultado se debería al azar (probabilidad de que la diferencia observada en el estudio se deba al azar). Por ende, cuanto mayor sea el valor de $\mathrm{p},(\mathrm{p}>0,1)$, más fuerte y segura será la evidencia a favor de la hipótesis nula (Altman).

OTZEN, T.; MANTEROLA, C.; RODRÍGUEZ-NÚÑEZ, I. \& GARCÍA-DOMÍNGUEZ, M. The scientific method. Its relevance in conducting clinical research. Int. J. Morphol.,35(3):1031-1036, 2017.

SUMMARY: Research methodology is a set of methods, laws and procedures that guide research efforts towards solving scientific problems with maximum efficiency. The method is based on the development of different stages that lead to the establishment of a valid conclusion supported on the verification of a hypothesis and assumptions of the performed research. Thus, scientific research is not exempt from the errors, which can originate during the study issue, research design, establishment of eligibility criteria, sample size estimation, measurement process, during follow-up or due to lack of discussion of study limitations. In this context, one of the strategies to reduce potential errors in conducting a study lies in the proper development of a research project or protocol, which must be elaborated based on a research, design and method problem. This provides the researcher with a number of advantages associated with documented process planning, reproducibility of experimental protocols and facilitation of the results publication. This review aims to discuss the essential aspects regarding the scientific method in clinical research and to analyze the main problems, benefits and elements associated with the feasibility of developing a research protocol.

KEY WORDS: "Epidemiologic Research Design"[Mesh]; "Research Design"[Mesh]; "Patient Selection"[Mesh]); "Methods"[Mesh]); "Methods/epidemiology"[Mesh].

\section{REFERENCIAS BILIOGRÁFICAS}

Altman, D. G. Statistics in medical journals: some recent trends. Stat. Med., 19(23):3275-89, 2000.

Antoja Ribó, F. La inocencia de las cigüeñas. Rev. Lab. Clin., 3(2):51, 2010.

Choh, V. \& Priolo, S. Relating clinical study design to basic research. Optom. Vis. Sci., 76(7):462-7, 1999.

Clark, G. T. \& Mulligan, R. Fifteen common mistakes encountered in clinical research. J. Prosthodont. Res., 55(1):1-6, 2011.

de Rovira, J. B. \& Tapia Granados, J. A. Mitos o realidades: a propósito de la publicación de trabajos científicos. ACIMED, 4(1):41-6, 1996.

Fava, G. A.; Tomba, E. \& Sonino, N. Clinimetrics: the science of clinical measurements. Int. J. Clin. Pract., 66(1):11-5, 2012.

Feinstein, A. R. An additional basic science for clinical medicine: IV. The development of clinimetrics. Ann. Intern. Med., 99(6):843-8, 1983.

Hartung, D. M. \& Touchette, D. Overview of clinical research design. Am. J. Health Syst. Pharm., 66(4):398-408, 2009.

Jiménez, R. Metodología de la Investigación. Elementos Básicos para la Investigación Clínica. La Habana, Editorial Ciencias Médicas, 1998.

Lader, E. W.; Cannon, C. P.; Ohman, E. M.; Newby, L. K.; Sulmasy, D. P.; Barst, R. J.; Fair, J. M.; Flather, M.; Freedman, J. E.; Frye, R. L.; Hand, M. M.; Jesse, R. L.; Van de Werf, F.; Costa, F. \& American Heart Association. The clinician as investigator: participating in clinical trials in the practice setting: Appendix 1: fundamentals of study design. Circulation, 109(21):e302-4, 2004.

Manterola, C. \& Otzen, H. T. Why research and how to conduct an research. Int. J. Morphol., 31(4):1498-504, 2013.

Manterola, C. \& Otzen, T. Bias in clinical research. Int. J. Morphol., 33(3):115664, 2015.

Manterola, C. \& Otzen, T. Checklist for reporting results using observational descriptive studies as research designs. The MInCir initiative. Int. J. Morphol., 35(1):72-6, 2017.

Manterola, C. \& Otzen, T. Observational studies. The most commonly used designs in clinical research. Int. J. Morphol., 32(2):634-45, 2014.

Manterola, C.; Asenjo-Lobos, C. \& Otzen, T. Hierarchy of evidence: levels of evidence and grades of recommendation from current use. Rev. Chil. Infectol., 31(6):705-18, 2014.

Manterola, D. C.; Pineda, N. V. \& Grupo Mincir. El valor de "p” y la "significación estadística". Aspectos generales y su valor en la práctica clínica. Rev. Chil. Cir., 60(1):86-9, 2008.

Pita Fernández, S. Dificultades de los médicos para la realización de estudios clínico-epidemiológicos. Ponencia. Santiago de Compostela, Congreso Galego de Estadística e Investigación de Operacións, 11 al 13 Noviembre de 1999, 1999. Disponible en: http://www.fisterra.com/mbe/investiga/2dificultades/ 2dificultades.asp

Pocock, S. J. The role of statistics in medical research. Br. J. Psychiatry, 137:18890, 1980.

Rubino, M. Importance of the scientific method in clinical research. Ann. Ital. Chir., 60(1):3-4, 1988

Sessler, D. I. \& Imrey, P. B. Clinical research methodology 1: Study designs and methodologic sources of error. Anesth. Analg., 121(4):1034-42, 2015.

Talavera, J. O. Clinical research I. The importance of the research design. Rev. Med. Inst. Mex. Seguro Soc., 49(1):53-8, 2011.

Temml, C. H. Research steps of applied medicine. Wien Med. Wochenschr., 151(78):170-2, 2001

Tomba, E. \& Bech, P. Clinimetrics and clinical psychometrics: macro- and microanalysis. Psychother. Psychosom., 81(6):333-43, 2012.

Dirección para correspondencia:

Dr. Carlos Manterola Delgado, CEMyQ

Universidad de La Frontera

Manuel Montt 112, Oficina 408

Temuco - CHILE

Recibido :11-05-2017

Aceptado:24-06-2017

E-mail: carlos.manterola@ufrontera.cl 